\section{Kidney \\ Blood Pressure Research}

\title{
High Neutrophil-to-Lymphocyte Ratio is a Significant Predictor of Cardiovascular and All-Cause Mortality in Patients Undergoing Peritoneal Dialysis
}

\author{
Xiangxue Lu ${ }^{\mathrm{a}}$ Shixiang Wang ${ }^{\mathrm{a}}$ Guizhi Zhang ${ }^{\mathrm{a}}$ Ruifang Xiong ${ }^{\mathrm{a}} \mathrm{Han} \mathrm{Li}^{\mathrm{a}}$ \\ aDepartment of Blood Purification, Beijing Chao-Yang Hospital, Capital Medical University, Beijing, \\ China
}

\section{Key Words}

Peritoneal dialysis - Neutrophil-to-lymphocyte ratio - Arterial stiffness - Cardiovascular mortality

\begin{abstract}
Background/Aims: Chronic inflammation is associated with increased risk of cardiovascular death in patients with end-stage renal disease (ESRD). Although elevated neutrophil-tolymphocyte ratio (NLR), a novel inflammatory marker, has been shown to predict cardiovascular disease and all-cause mortality in the general population, limited evidence is available for its role in ESRD. Methods: We enrolled 86 patients undergoing peritoneal dialysis (PD) for a 36-month follow-up to investigate the association between the NLR and arterial stiffness markers, namely, carotid-femoral pulse wave velocity (cfPWV) and carotid augmentation index (AIx), and mortality in PD patients. The primary endpoints were cardiovascular mortality and all-cause mortality. Kaplan-Meier curves were used to show the cumulative incidence of cardiovascular mortality and all-cause mortality. Results: High NLR was found to be a predictor of increased cfPWV $(\beta=1.150 ; P<0.001)$ and AIx $(\beta=3.945 ; P<0.001)$ in patients on PD. Patients with higher NLR had lower survival during follow-up. Kaplan-Meier curves showed that the cumulative incidences of both cardiovascular mortality and all-cause mortality were significantly higher in patients with NLR $\geq 4.5$ (both $P<0.01$ ). Conclusion: Our results suggest that high NLR is independently associated with arterial stiffness and predicts cardiovascular and all-cause mortality in PD patients.




\section{Kidney Blood Pressure Research}

Lu et al.: NLR and Mortality in Patients on Peritoneal Dialysis

\section{Introduction}

Cardiovascular disease is the main complication and major cause of death in patients undergoing peritoneal dialysis (PD), accounting for nearly $60 \%$ of all deaths in this population [1]. The high cardiovascular mortality associated with chronic renal disease, especially in patients with end-stage renal disease (ESRD), is not entirely explained by traditional cardiovascular risk factors [2]. Microinflammation is a key factor in the malnutritioninflammation-atherosclerosis/calcification syndrome, which further accelerates the progression of atherosclerosis and is associated with increased risk of cardiovascular disease in patients on PD [3].

Neutrophil-to-lymphocyte ratio (NLR) is obtained simply by dividing the absolute neutrophil count by the absolute lymphocyte count in peripheral blood. Recently, NLR has been recognized as a novel inflammatory marker for assessing cardiovascular disease severity and poor prognosis in the general population [4] and in patients with ESRD [5, 6].

Increased arterial stiffness, an early marker of atherosclerosis, has been shown to be a powerful independent predictor of cardiovascular events and all-cause mortality in patients with chronic kidney disease (CKD) [7]. Carotid-femoral pulse wave velocity (cfPWV) and augmentation index (AIx) are widely used as surrogate markers of arterial stiffness [8]. An increasing number of studies have demonstrated that increased cfPWV is associated with increased risk of renal disease and incident cardiovascular events, as well as increased allcause mortality [9-12].

However, to date there has been little evidence to show an association between NLR and arterial stiffness in patients on PD, and the prognostic value of NLR in this population is not yet entirely clear. Therefore, in this study, we evaluated the relationship between NLR and arterial stiffness markers, including cfPWV and AIx, and further investigated the association between increased NLR and mortality in patients on PD.

\section{Materials and Methods}

\section{Data sources}

One hundred five patients with ESRD receiving PD treatment in the Department of Blood Purification, Beijing Chao-Yang Hospital, Capital Medical University were recruited from January 2014 through December 2016. Inclusion criteria were ESRD with no residual renal function and regular PD treatment for at least 3 months. Exclusion criteria were clinical evidence of heart failure; a recent acute coronary or cerebrovascular event; autoimmune disease, malignancy or active infection; and medication history of aspirin, statins, steroids, or immunosuppressive drugs. Nineteen patients were excluded for the following reasons: clinical evidence of heart failure $(n=4)$, a recent acute coronary event $(n=3)$, a recent cerebrovascular event $(n=2)$, history of malignancy $(n=3)$, history of autoimmune disease $(n=3)$, clinical evidence of active infection $(n=2)$, and history of aspirin use $(n=2)$. The remaining 86 patients were undergoing continuous ambulatory PD treatment performed using Baxter Twin Bag (Baxter Healthcare, Guangzhou, China) with a daily dialysis dose of 6-8 L.

Patients were followed for 36 months. The primary endpoints were cardiovascular mortality and all-cause mortality. Cardiovascular death included death caused by coronary events, arrhythmias, sudden cardiac death, congestive heart failure, and cerebrovascular events [13]. The study was performed according to the Declaration of Helsinki and approved by the ethics committee of Beijing Chao-Yang Hospital, Capital Medical University. Written informed consent was obtained from each participant.

\section{Biochemical measurements and calculation of NLR}

Blood samples from study patients were taken after a 10-h overnight fast prior to dialysis. The blood samples were drawn into plastic vacutainers with EDTA (1 mg/mL of blood) for differential white blood cell 


\section{Kidney Blood Pressure Research}

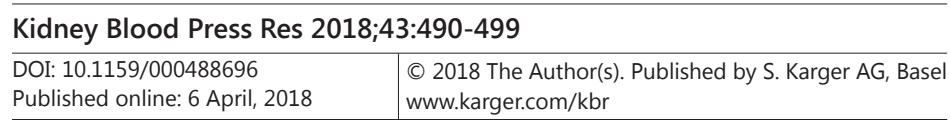

Lu et al.: NLR and Mortality in Patients on Peritoneal Dialysis

(WBC) count. NLR was calculated as the ratio of neutrophils to lymphocytes. Blood chemistry parameters were assayed by standardized and automated techniques in the same laboratory.

Arterial stiffness measurement

Common carotid artery stiffness was evaluated by cfPWV and AIx using the Complior SP System (Alam Medical, Vincennes, France) [14, 15], with participants in a supine position.

\section{Carotid intima-media thickness measurement}

Carotid intima-media thickness (IMT) was evaluated by carotid artery ultrasonography as described previously [16]. The presence of carotid plaque was defined as localized thickening (IMT $\geq 1.2 \mathrm{~mm}$ ) that did not uniformly involve the whole wall of the carotid artery.

\section{Statistical analysis}

All data were analyzed using SPSS for Windows (Version 20.0, IBM Corp, Armonk, NY, USA). Continuous variables were presented as mean \pm standard deviation $( \pm \mathrm{SD})$ or median (25th-75th percentile). Student's t tests, chi-square tests, or Mann-Whitney U tests were used to compare variables between groups, as appropriate. In addition, Spearman correlation was used for univariate analysis and logistic regression was used for multivariate analysis (confidence interval of 95\%). Survival curves were estimated by Kaplan-Meier analysis and compared using the log-rank test. P values $<0.05$ were considered statistically significant.

\section{Results}

\section{Patient characteristics}

A total of 86 patients were enrolled in the study. Mean patient age was $54.6 \pm 14.0$ years (range 23-79 years) and the mean dialysis duration was $25.6 \pm 11.2$ months (range 7-51 months). The primary causes of ESRD were chronic glomerulonephritis (39.5\%), diabetic nephropathy (17.4\%), chronic interstitial nephritis (12.8\%), hypertensive nephropathy (9.3\%), and unknown causes (20.9\%). Baseline patient characteristics are shown in Table 1.
Table 1. Baseline patient characteristics. Values are means \pm SD or median (25th-75th percentile), unless specified otherwise. NLR = neutrophil-to-lymphocyte ratio; $\mathrm{cfPWV}=$ carotidfemoral pulse wave velocity; $\mathrm{AIx}=$ augmentation index; $\mathrm{Hb}=$ hemoglobin; Alb = albumin; ALT = alanine transaminase; AST = aspartate aminotransferase $\mathrm{TG}=$ triglyceride; $\mathrm{Tch}=$ total cholesterol; LDL-C = low-density lipoprotein cholesterol; hsCRP = high-sensitivity C-reactive protein; $\mathrm{Scr}=$ serum creatinine; BUN = blood urea nitrogen; $\mathrm{Ca}=$ calcium; $\mathrm{P}=$ phosphorus; $\mathrm{iPTH}=$ intact parathyroid hormone; RASI = renin angiotensin system inhibitor; $\mathrm{CCB}=$ calcium channel blocker; $\beta$-blocker $=$ $\beta$-receptor blocker

\begin{tabular}{lc}
\hline Items & Patients (n=86) \\
\hline Age, years & $54.6 \pm 14.0$ \\
Sex, male, $\mathrm{n}(\%)$ & $37(43.0 \%)$ \\
Primary disease & \\
\multicolumn{1}{c}{ Chronic glomerulonephritis, n (\%) } & $34(39.5 \%)$ \\
Diabetic nephropathy, $\mathrm{n}(\%)$ & $15(17.4 \%)$ \\
Chronic interstitial nephritis, n (\%) & $11(12.8 \%)$ \\
$\quad$ Hypertensive nephropathy, n (\%) & $8(9.3 \%)$ \\
$\quad$ Unknown causes, $\mathrm{n}(\%)$ & $18(20.9 \%)$ \\
PD duration, months & $25.6 \pm 11.2$ \\
Smoking, n (\%) & $9(10.5 \%)$ \\
Diabetes, n (\%) & $16(18.6 \%)$ \\
NLR & $4.50(2.50-5.97)$ \\
cfPWV, mm/s & $10.1 \pm 3.5$ \\
AIx, \% & $29.5 \pm 17.6$ \\
Carotid plaque, n (\%) & $34(49.3 \%)$ \\
Hb, g/L & $114.6 \pm 10.9$ \\
Alb, g/L & $35.5 \pm 3.3$ \\
ALT, U/L & $17.4 \pm 6.0$ \\
AST, U/L & $19.2 \pm 6.8$ \\
TG, mmol/L & $1.74 \pm 0.79$ \\
Tch, mmol/L & $4.57 \pm 0.76$ \\
LDL-C, mmol/L & $2.91 \pm 0.97$ \\
hsCRP, mmol/L & $1.83(1.49-4.11)$ \\
Scr, $\mu$ mol/L & $888.4 \pm 173.6$ \\
BUN, mmol/L & $21.6 \pm 6.3$ \\
Ca, mmol/L & $2.20 \pm 0.24$ \\
P, mmol/L & $1.46 \pm 0.32$ \\
iPTH, pg/ml & $207.5(147.3-250.0)$ \\
RASI, n (\%) & $44(63.8 \%)$ \\
CCB, n (\%) & $54(78.3 \%)$ \\
$\beta$-blocker, $n(\%)$ & $24(34.8 \%)$ \\
\hline
\end{tabular}




\section{Kidney Blood Pressure Research}

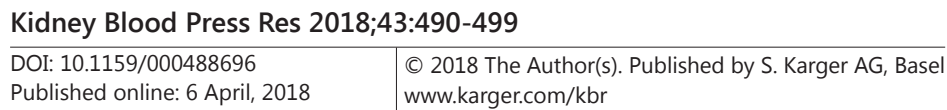

Lu et al.: NLR and Mortality in Patients on Peritoneal Dialysis

Table 2. Characteristics of PD patients with and without carotid plaque. Values are means \pm SD or median (25th-75th percentile), unless specified otherwise. ${ }^{a} P<0.01$, compared with non-plaque group. NLR $=$ neutrophil-to-lymphocyte ratio; $\mathrm{cfPWV}=$ carotid-femoral pulse wave velocity; $\mathrm{AIx}=$ augmentation index; $\mathrm{Hb}=$ hemoglobin; Alb = albumin; ALT = alanine transaminase; $\mathrm{AST}=$ aspartate aminotransferase; $\mathrm{TG}=$ triglyceride; Tch = total cholesterol; LDL-C = low-density lipoprotein cholesterol; hsCRP = high-sensitivity C-reactive protein; Scr=serum creatinine; $\mathrm{BUN}=$ blood urea nitrogen; $\mathrm{Ca}=$ calcium; $\mathrm{P}=$ phosphorus; $\mathrm{PTH}=$ parathyroid hormone; RASI = renin angiotensin system inhibitor; $\mathrm{CCB}=$ calcium channel blocker; $\beta$-blocker $=\beta$-receptor blocker

\begin{tabular}{|c|c|c|c|}
\hline Items & PD without carotid plaque group $(n=42)$ & PD with carotid plaque & $\mathrm{P}$ value \\
\hline Age, years & $55.7 \pm 13.9$ & $53.6 \pm 14.2$ & 0.499 \\
\hline Sex, male/female & $18 / 24$ & $19 / 25$ & 0.976 \\
\hline PD duration, months & $26.0 \pm 10.8$ & $25.3 \pm 11.7$ & 0.749 \\
\hline BMI, $\mathrm{kg} / \mathrm{m}^{2}$ & $24.0 \pm 2.4$ & $24.5 \pm 3.0$ & 0.409 \\
\hline Smoking, no.(\%) & $5(11.9)$ & $4(9.1)$ & 0.670 \\
\hline Diabetes, no. (\%) & 7 (16.7) & $9(20.5)$ & 0.652 \\
\hline NLR & $2.96(2.33-5.40)$ & $5.07(3.45-6.47)^{\mathrm{a}}$ & 0.012 \\
\hline $\mathrm{cfPWV}, \mathrm{mm} / \mathrm{s}$ & $8.9 \pm 2.9$ & $11.3 \pm 3.7$ a & 0.001 \\
\hline AIx, \% & $24.2 \pm 15.8$ & $34.5 \pm 17.9$ a & 0.006 \\
\hline $\mathrm{Hb}, \mathrm{g} / \mathrm{L}$ & $114.7 \pm 11.8$ & $114.6 \pm 10.4$ & 0.921 \\
\hline Alb, g/L & $35.6 \pm 3.4$ & $35.4 \pm 3.2$ & 0.729 \\
\hline ALT, U/L & $17.4 \pm 5.4$ & $17.5 \pm 6.6$ & 0.928 \\
\hline AST, U/L & $18.3 \pm 5.7$ & $20.1 \pm 7.7$ & 0.223 \\
\hline $\mathrm{TG}, \mathrm{mmol} / \mathrm{L}$ & $1.78 \pm 0.90$ & $1.70 \pm 0.68$ & 0.643 \\
\hline $\mathrm{Tch}, \mathrm{mmol} / \mathrm{L}$ & $4.52 \pm 0.73$ & $4.61 \pm 0.80$ & 0.574 \\
\hline LDL-C, mmol/L & $2.92 \pm 1.10$ & $2.90 \pm 0.84$ & 0.946 \\
\hline hsCRP, mmol/L & $1.74(1.15-1.93)$ & $3.99(1.60-5.10)^{\mathrm{a}}$ & 0.005 \\
\hline $\mathrm{Scr}, \mu \mathrm{mol} / \mathrm{L}$ & $853.9 \pm 163.2$ & $893.8 \pm 153.2$ & 0.222 \\
\hline BUN, mmol/L & $21.7 \pm 6.7$ & $21.5 \pm 5.9$ & 0.855 \\
\hline $\mathrm{Ca}, \mathrm{mmol} / \mathrm{L}$ & $2.20 \pm 0.26$ & $2.20 \pm 0.22$ & 0.986 \\
\hline $\mathrm{P}, \mathrm{mmol} / \mathrm{L}$ & $1.50 \pm 0.35$ & $1.43 \pm 0.28$ & 0.325 \\
\hline PTH, pg/ml & $204.0(145.0-253.0)$ & $228.5(150.0-257.0)$ & 0.447 \\
\hline RASI, no.(\%) & $26(61.9)$ & $31(70.5)$ & 0.402 \\
\hline CCB, no. $(\%)$ & $32(76.2)$ & $36(81.8)$ & 0.521 \\
\hline$\beta$-blocker, no. $(\%)$ & $14(33.3)$ & $11(25.0)$ & 0.395 \\
\hline
\end{tabular}

Characteristics of PD patients with and without common carotid artery plaque

Forty-four of 86 patients (51.2\%) had plaque in the common carotid artery as assessed by IMT. NLR was significantly higher in patients with carotid plaque than in patients without carotid plaque. Interestingly, cfPWV and AIx were also higher in patients with carotid plaque. However, there were no significant differences with respect to age, sex distribution, PD duration, body mass index, diabetes, smoking, hemoglobin, serum creatinine, blood urea nitrogen, triglycerides, total cholesterol, low-density lipoprotein cholesterol (LDL-C), or highsensitivity C-reactive protein (hsCRP) between patients with and without carotid plaque (Table 2).

\section{Correlation of NLR with arterial stiffness markers in $P D$ patients}

Correlations between NLR and patient characteristics are shown in Table 3. NLR was positively correlated with cfPWV ( $\mathrm{r}=0.857 ; P<0.001)$, AIx $(\mathrm{r}=$ $0.609 ; P<0.001)$, and hsCRP $(\mathrm{r}=0.515 ; P<0.001)$. NLR was not significantly correlated with age, sex, PD duration, smoking, diabetes, or serum LDL-C.

In the multivariate analysis, NLR was independently correlated with cfPWV $(\beta=1.150$; $P<0.001)$ and AIx $(\beta=3.945 ; P<0.001)$ (Table 4).
Table 3. Correlation analysis for variables and NLR in PD patients. $\mathrm{cfPWV}=$ carotid-femoral pulse wave velocity; AIx = augmentation index; LDL-C = low-density lipoprotein C-reactive protein

\begin{tabular}{lcc}
\hline Variables & $\mathrm{r}$ & P value \\
\hline Age, years & 0.102 & 0.352 \\
Sex & 0.154 & 0.156 \\
PD duration, months & 0.088 & 0.420 \\
Smoking & 0.027 & 0.807 \\
Diabetes & 0.030 & 0.783 \\
LDL-C, mmol/L & 0.128 & 0.241 \\
hsCRP, mmol/L & 0.515 & $<0.001$ \\
cfPWV, mm/s & 0.857 & $<0.001$ \\
AIx, \% & 0.609 & $<0.001$ \\
\hline
\end{tabular}
cholesterol; hsCRP = high-sensitivity 


\section{Kidney Blood Pressure Research}

Table 4. Multivariate associations of cfPWV and AIx in PD patients. NLR = neutrophil-to-lymphocyte ratio; cfPWV = carotid-femoral pulse wave velocity; AIx = augmentation index; LDL-C= low-density lipoprotein cholesterol; hsCRP = high-sensitivity C-reactive protein

\begin{tabular}{lcccccc}
\hline \multirow{2}{*}{ Variables } & \multicolumn{2}{c}{ cfPWV } & & \multicolumn{2}{c}{ AIx } & P value \\
\hline Age & $\beta$ coefficient & $\mathrm{t}$ & P value & $\beta$ coefficient & $\mathrm{t}$ & 0.498 \\
Sex & 0.018 & 1.102 & 0.274 & 0.053 & 0.310 & 0.757 \\
PD duration & 0.239 & 0.532 & 0.596 & 0.931 & 0.664 & 0.509 \\
Smoking & 0.012 & 0.617 & 0.539 & 0.089 & 0.027 & 0.978 \\
Diabetes & 1.101 & 1.513 & 0.134 & 0.132 & 1.107 & 0.272 \\
NLR & 0.372 & 0.622 & 0.536 & 4.428 & 4.126 & $<0.001$ \\
LDL-C & 1.150 & 8.030 & $<0.001$ & 3.945 & 0.126 & 0.900 \\
hsCRP & 0.377 & 1.533 & 0.129 & 0.207 & 2.797 & 0.007 \\
\hline
\end{tabular}

Fig. 1. Box plots for NLR levels in $\mathrm{PD}$ patients with all-cause and cardiovascular death. A. PD patients who died from all causes had significantly higher NLR levels $(P<0.01)$; B. PD patients who died from cardiovascular causes had significantly higher NLR levels $(P$ $<0.01$ ).
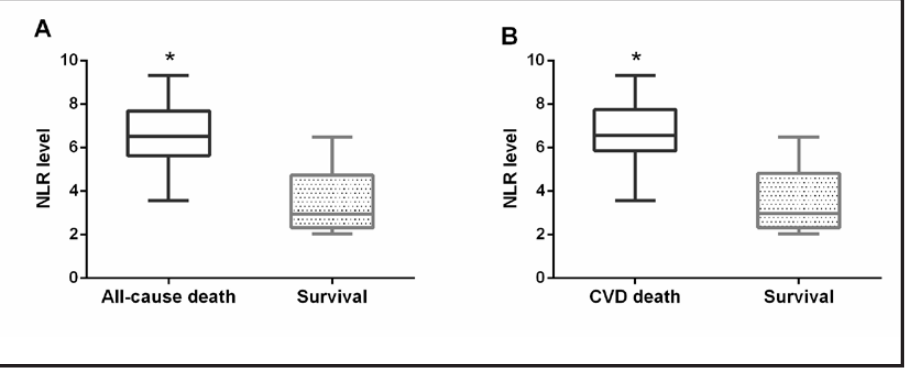

Fig. 2. Kaplan-Meier plots for allcause mortality and cardiovascular mortality in PD patients. A. Patients with NLR $\geq 4.5$ had significantly higher 36-month all-cause mortality $(\log$ rank $=25.162 ; P<$ 0.01 ); B. Patients with NLR $\geq 4.5$ had significantly higher 36-month cardiovascular mortality (log rank = 25.530; $P<0.01$ ).

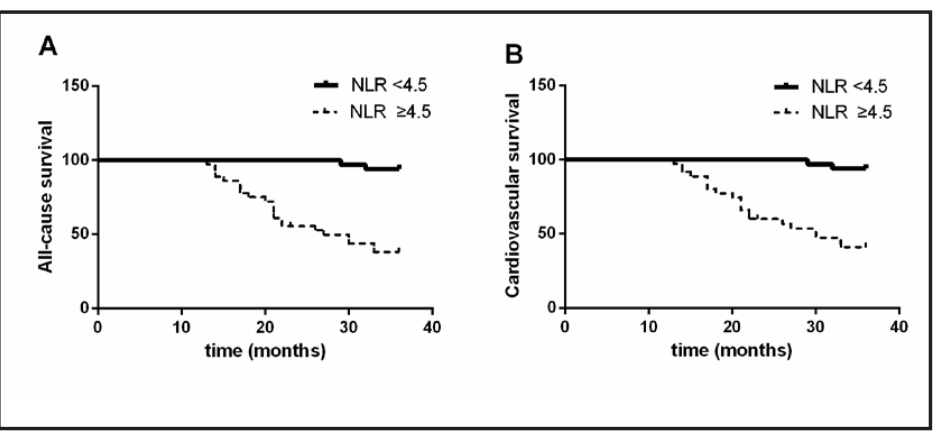

NLR levels in PD patients with cardiovascular death and all-cause death

Of the 37/86 patients (43.0\%) who died during the study period, 24 (64.9\%) died of cardiovascular events. NLR was higher in patients who died of any cause vs those who survived (6.09 [4.99-7.56] vs 2.92 [2.30-4.65]; $P<0.01$ ), as well as in patients who died of cardiovascular causes vs those who survived (6.57 [5.94-7.73] vs 2.98 [2.33-4.90]; $P<0.01$ ) (Fig. $1 \mathrm{~A}$ and B).

\section{Association of NLR with all-cause and cardiovascular death in PD patients}

When patients were divided into 2 groups depending on median NLR (below or above the median value of 4.5), overall survival (log rank $=25.162 ; P<0.01$ ) and cardiovascular survival (log rank = 25.530; $P<0.01$ ) were significantly lower in the group with higher NLR (Fig. $2 \mathrm{~A}$ and $\mathrm{B}$ ). 


\section{Kidney Blood Pressure Research}

Kidney Blood Press Res 2018;43:490-499

\begin{tabular}{l|l}
\hline DOI: 10.1159/000488696 & (C) 2018 The Author(s). Published by S. Karger AG, Basel
\end{tabular}

Published onlıne: 6 April, 2018

www.karger.com/kb

Lu et al.: NLR and Mortality in Patients on Peritoneal Dialysis

\section{Discussion}

The results of this study demonstrate that elevated NLR is associated with arterial stiffness in patients undergoing PD. Multiple linear regression analysis showed that NLR is an independent factor for increased cfPWV and AIx. Interestingly, we further found that elevated NLR was a significant predictor of all-cause mortality and cardiovascular mortality in PD patients, independent of clinical, biochemical, and inflammatory parameters.

These findings are similar to those previously reported in the general population and in patients with ESRD, in which elevated NLR as a novel inflammation marker has been shown to be strongly and independently predictive of cardiovascular disease severity and mortality. In patients with severe calcific aortic stenosis, NLR was the independent prognostic factor most significantly associated with major adverse cardiovascular events [17]. In patients with pulmonary arterial hypertension, NLR was also useful for assessment of disease severity [18]. In patients with obstructive sleep apnea syndrome, NLR was independently associated with cardiovascular disease and was a predictor of disease severity [19]. Patients with type 2 diabetes who had high NLR would be more vulnerable to significant coronary artery disease and carotid artery atherosclerosis [20]. In medical inpatients with multiple chronic conditions, increased NLR was associated with mortality [21]. In patients with peripheral arterial occlusive disease, increased NLR was related with higher cardiovascular mortality [22]. Okyay et al. [23] noted that laboratory measurement of NLR, which is relatively easy and inexpensive, might provide significant information regarding inflammation in CKD, including in predialysis and dialysis patients. Malhotra et al. [24] suggested that NLR could serve as a potential surrogate marker for CRP in hemodialysis patients. Similarly, Ahbap et al. [6] found that NLR was significantly positively correlated with hsCRP levels in patients with ESRD on maintenance hemodialysis. In patients on prevalent hemodialysis, NLR was also associated with all-cause mortality [25]. We recently reported that NLR was also associated with allcause and cardiovascular mortality in patients on hemodialysis [26]. NLR was found to be an independent predictor of left atrium mechanical function and atrial electromechanical delay times in patients with ESRD receiving peritoneal dialysis or hemodialysis [27]. Turkmen et al. [5] also found that NLR could predict vascular calcification in patients with ESRD receiving peritoneal dialysis or hemodialysis. Recently, Cai et al. [1] reported that NLR was independently associated with brachial-ankle pulse wave velocity (baPWV) in patients on peritoneal dialysis. But to date, little was known of the prognostic value of NLR in patients undergoing PD. Although An et al. [28] reported in 2012 that NLR was a strong predictor for overall and cardiovascular mortality in peritoneal dialysis patients, they did not investigate the association between NLR and arterial stiffness. In the present study, we investigated the association between NLR and arterial stiffness markers, namely, cfPWV and AIx, and mortality in PD patients.

Cardiovascular disease is the leading cause of death in peritoneal dialysis patients [29]. Traditional risk factors such as hypertension, diabetes, hyperlipidemia, smoking, and male sex do not explain the abnormally high incidence of cardiovascular disease in this patient population. Inflammation, which is a central process in the development of cardiovascular disease, is prevalent in patients with CKD [30]. Patients affected by different stages of CKD, particularly those on hemodialysis, present with a marked activation of inflammatory processes, which exposes them to elevated risk of morbidity and mortality [31]. High levels of free radicals have been reported in the course of renal failure, while patients with advanced CKD accumulate low molecular weight toxins with pro-oxidant activity [32]. So far, many plasma inflammation markers of cardiovascular disease, such as CRP and interleukin-6, have been found to have great predictive value and applied to clinical practice [33]. However, measurement of these markers is relatively expensive and not easy to conduct on a widespread basis. NLR, which is obtained by dividing the absolute neutrophil count by the absolute lymphocyte count, is an emerging marker for assessing inflammation and has been found to be of great predictive value for cardiovascular disease. 


\section{Kidney Blood Pressure Research}

Kidney Blood Press Res 2018;43:490-499
\begin{tabular}{l|l} 
DOI: 10.1159/000488696 & $\begin{array}{l}\text { ( ) 2018 The Author(s). Published by S. Karger AG, Basel } \\
\text { www.karger.com/kbr }\end{array}$ \\
Published onlIne: 6 April, 2018 &
\end{tabular}

Lu et al.: NLR and Mortality in Patients on Peritoneal Dialysis

Yombi et al. [34] found that NLR levels return to normal more quickly than CRP levels in a standard postoperative period after total knee arthroplasty. A recent study conducted by Yilmaz et al. indicated that NLR was better than CRP for predicting the occurrence of osteoporosis [35]. Several potential mechanisms may explain the association between NLR and cardiovascular disease: neutrophils are proinflammatory cells, which could aggravate endothelial dysfunction, activate macrophages, and promote foam cell formation [36]; meanwhile, lymphopenia is associated with the progression of atherosclerosis and adverse cardiovascular outcomes, which may be due to the apoptosis of lymphocytes [37]. NLR may therefore increase in several cardiovascular diseases, and it is more stable than individual WBC counts. Based on our research, NLR is a potentially effective and inexpensive predictor of cardiovascular and all-cause mortality in peritoneal dialysis patients. Arterial stiffness, a novel cardiovascular risk factor, has been shown as a strong and independent predictor of cardiovascular disease in patients with kidney disease [38]. cfPWV and AIx, markers of arterial stiffness, are considered independent predictors of major cardiovascular events [39]. To our knowledge, the present study is the first to examine the relationship of NLR with cfPWV and AIx. Although some studies have shown that cfPWV and baPWV exhibit similar associations with cardiovascular disease risk factors and clinical events in the general population [40], its applicability to patients on PD is still controversial. For example, one study showed that baPWV was not useful for risk stratification of systemic atherosclerotic morbidity and mortality in patients on hemodialysis [41]. Markers of arterial stiffness such as cfPWV and AIx are widely used to predict coronary artery disease and cardiovascular disease, especially in PD patients [42, 43]. NLR, which is obtained by dividing the absolute neutrophil count by the absolute lymphocyte count, is easily calculated from differential WBC counts, more stable for measurement than individual WBC counts, and less affected by conditions that could change individual cell counts. According to our findings, an easy and inexpensive laboratory measurement of NLR might provide significant information regarding arterial stiffness markers, including cfPWV and AIx, to help predict cardiovascular disease and all-cause mortality in patients undergoing PD.

However, there were some limitations to our study. First, this was a non-randomized, single-center study that included a relatively small number of patients, which may lead to selection bias. Second, we did not compare NLR with other inflammatory markers such as $\mathrm{CRP}$, and therefore cannot assess their relative predictive value for cardiovascular disease.

\section{Conclusion}

This study demonstrated that increased NLR was independently associated with a higher risk of arterial stiffness and higher all-cause and cardiovascular death in peritoneal dialysis patients. NLR, which is easy to assess, may be a novel marker of cardiovascular disease in this patient population. Further research is required to elucidate the mechanism underlying the relationship between NLR and cardiovascular disease, and to identify effective antiinflammatory treatments to prevent cardiovascular disease in peritoneal dialysis patients.

\section{Acknowledgements}

This work was supported by the National Natural Science Foundation of China (81670673) and the Beijing Natural Science Foundation (7182060).

\section{Disclosure Statement}

The authors declare no conflict of interest. 


\section{Kidney \\ Blood Pressure Research}

\begin{tabular}{l}
\hline Kidney Blood Press Res 2018;43:490-499 \\
\begin{tabular}{l|l}
\hline DOI: 10.1159/000488696 \\
Published onlIne: 6 April, 2018
\end{tabular} \\
\hline
\end{tabular}

Lu et al.: NLR and Mortality in Patients on Peritoneal Dialysis

\section{References}

1 Cai K, Luo Q, Zhu B, Han L, Wu D, Dai Z, Wang K: Neutrophil-lymphocyte ratio is associated with arterial stiffness in patients with peritoneal dialysis. BMC Nephrol 2016;17:191.

-2 Tonelli M, Karumanchi SA, Thadhani R: Epidemiology and Mechanisms of Uremia-Related Cardiovascular Disease. Circulation 2016;133:518-536.

- Neirynck N, Glorieux G, Schepers E, Dhondt A, Verbeke F, Vanholder R: Pro-inflammatory cytokines and leukocyte oxidative burst in chronic kidney disease: culprits or innocent bystanders? Nephrol Dial Transplant 2015;30:943-951.

-4 Argan O, Ural D, Kozdag G, Sahin T, Bozyel S, Aktas M, Karauzum K, Yilmaz I, Dervis E, Agir A: Associations Between Neutrophil Gelatinase Associated Lipocalin, Neutrophil-to-Lymphocyte Ratio, Atrial Fibrillation and Renal Dysfunction in Chronic Heart Failure. Med Sci Monit 2016;22:4765-4772.

5 Turkmen K, Ozcicek F, Ozcicek A, Akbas EM, Erdur FM, Tonbul HZ: The relationship between neutrophil-tolymphocyte ratio and vascular calcification in end-stage renal disease patients. Hemodial Int 2014;18:4753.

6 Ahbap E, Sakaci T, Kara E, Sahutoglu T, Koc Y, Basturk T, Sevinc M, Akgol C, Kayalar AO, Ucar ZA, Bayraktar F, Unsal A: Neutrophil-to-lymphocyte ratio and platelet-tolymphocyte ratio in evaluation of inflammation in end-stage renal disease. Clin Nephrol 2016;85:199-208.

7 Karras A, Haymann JP, Bozec E, Metzger M, Jacquot C, Maruani G, Houillier P, Froissart M, Stengel B, Guardiola P, Laurent S, Boutouyrie P, Briet M, Nephro Test Study G: Large artery stiffening and remodeling are independently associated with all-cause mortality and cardiovascular events in chronic kidney disease. Hypertension 2012;60:1451-1457.

8 Tuttolomondo A, Casuccio A, Guercio G, Maida C, Del Cuore A, Di Raimondo D, Simonetta I, Di Bona D, Pecoraro R, Della Corte V, Gulotta E, Gulotta G, Pinto A: Arterial stiffness, endothelial and cognitive function in subjects with type 2 diabetes in accordance with absence or presence of diabetic foot syndrome. Cardiovasc Diabetol 2017;16:2.

-9 Michener KH, Mitchell GF, Noubary F, Huang N, Harris T, Andresdottir MB, Palsson R, Gudnason V, Levey AS: Aortic stiffness and kidney disease in an elderly population. Am J Nephrol 2015;41:320-328.

10 Kong X, Ma X, Tang L, Wang Z, Li W, Cui M, Xu D: Arterial stiffness evaluated by carotid-femoral pulse wave velocity increases the risk of chronic kidney disease in a Chinese population-based cohort. Nephrology (Carlton) 2017;22:205-212.

11 van Sloten TT, Sedaghat S, Laurent S, London GM, Pannier B, Ikram MA, Kavousi M, Mattace-Raso F, Franco $\mathrm{OH}$, Boutouyrie P, Stehouwer CD: Carotid stiffness is associated with incident stroke: a systematic review and individual participant data meta-analysis. J Am Coll Cardiol 2015;66:2116-2125.

12 van Sloten TT, Schram MT, van den Hurk K, Dekker JM, Nijpels G, Henry RM, Stehouwer CD: Local stiffness of the carotid and femoral artery is associated with incident cardiovascular events and all-cause mortality: the Hoorn study. J Am Coll Cardiol 2014;63:1739-1747.

13 Liu X, Guo Q, Feng X, Wang J, Wu J, Mao H, Huang F, Yu X, Yang X: Alkaline phosphatase and mortality in patients on peritoneal dialysis. Clin J Am Soc Nephrol 2014;9:771-778.

14 Stea F, Bozec E, Millasseau S, Khettab H, Boutouyrie P, Laurent S: Comparison of the complior analyse device with sphygmocor and Complior SP for pulse wave velocity and central pressure assessment. J Hypertens 2014;32:873-880.

15 Maloberti A, Cesana F, Hametner B, Dozio D, Villa P, Hulpke-Wette M, Schwarz A, Selicorni A, Wassertheurer S, Mancia G, Giannattasio C: Increased nocturnal heart rate and wave reflection are early markers of cardiovascular disease in Williams-Beuren syndrome children. J Hypertens 2015;33:804-809.

-16 Li H, Feng SJ, Zhang GZ, Wang SX: Correlation of lower concentrations of hydrogen sulfide with atherosclerosis in chronic hemodialysis patients with diabetic nephropathy. Blood Purif 2014;38:188-194.

17 Cho KI, Cho SH, Her AY, Singh GB, Shin ES: Prognostic utility of neutrophil-to-lymphocyte ratio on adverse clinical outcomes in patients with severe calcific aortic stenosis. PLoS One 2016;11:e0161530.

18 Ozpelit E, Akdeniz B, Ozpelit ME, Tas S, Bozkurt S, Tertemiz KC, Sevinc C, Badak O: Prognostic value of neutrophil-to-lymphocyte ratio in pulmonary arterial hypertension. J Int Med Res 2015;43:661-671. 


\section{Kidney \\ Blood Pressure Research}

19 Uygur F, Tanriverdi H, Aktop Z, Erboy F, Altinsoy B, Damar M, Atalay F: The neutrophil-to-lymphocyte ratio in patients with obstructive sleep apnoea syndrome and its relationship with cardiovascular disease. Heart Lung 2016;45:121-125.

20 Kim BJ, Cho SH, Cho KI, Kim HS, Heo JH, Cha TJ: The Combined Impact of Neutrophil-to-Lymphocyte Ratio and Type 2 Diabetic Mellitus on Significant Coronary Artery Disease and Carotid Artery Atherosclerosis. J Cardiovasc Ultrasound 2016;24:115-122.

-21 Isaac V, Wu CY, Huang CT, Baune BT, Tseng CL, McLachlan CS: Elevated neutrophil to lymphocyte ratio predicts mortality in medical inpatients with multiple chronic conditions. Medicine (Baltimore) 2016;95:e3832.

-22 Erturk M, Cakmak HA, Surgit O, Celik O, Aksu HU, Akgul O, Gurdogan M, Bulut U, Ozalp B, Akbay E, Yildirim A: Predictive value of elevated neutrophil to lymphocyte ratio for long-term cardiovascular mortality in peripheral arterial occlusive disease. J Cardiol 2014;64:371-376.

23 Okyay GU, Inal S, Onec K, Er RE, Pasaoglu O, Pasaoglu H, Derici U, Erten Y: Neutrophil to lymphocyte ratio in evaluation of inflammation in patients with chronic kidney disease. Ren Fail 2013;35:29-36.

24 Malhotra R, Marcelli D, von Gersdorff G, Grassmann A, Schaller M, Bayh I, Scatizzi L, Etter M, Guinsburg A, Barth C, Marelli C, Carioni P, van der Sande F, Kooman JP, Liu E, Toffelmire T, Wang Y, Thijssen S, Usvyat LA, Kotanko P: Relationship of Neutrophil-to-Lymphocyte Ratio and Serum Albumin Levels with C-Reactive Protein in Hemodialysis Patients: Results from 2 International Cohort Studies. Nephron 2015;130:263-270.

-25 Yaprak M, Turan MN, Dayanan R, Akin S, Degirmen E, Yildirim M, Turgut F: Platelet-to-lymphocyte ratio predicts mortality better than neutrophil-to-lymphocyte ratio in hemodialysis patients. Int Urol Nephrol 2016;48:1343-1348.

-26 Li H, Lu X, Xiong R, Wang S: High Neutrophil-to-Lymphocyte Ratio Predicts Cardiovascular Mortality in Chronic Hemodialysis Patients. Mediators Inflamm 2017;2017:9327136.

-27 Demirtas L, Turkmen K, Buyuklu M, Kocyigit I, Orscelik 0: Atrial electromechanical delay and left atrial mechanical functions in hemodialysis and peritoneal dialysis patients. Int Urol Nephrol 2016;48:781-789.

-28 An X, Mao HP, Wei X, Chen JH, Yang X, Li ZB, Yu XQ Li ZJ: Elevated neutrophil to lymphocyte ratio predicts overall and cardiovascular mortality in maintenance peritoneal dialysis patients. Int Urol Nephrol 2012;44:1521-1528.

29 Shang D, Xie Q, Shang B, Zhang M, You L, Hao CM, Zhu T: Hyperphosphatemia and hs-CRP Initiate the Coronary Artery Calcification in Peritoneal Dialysis Patients. Biomed Res Int 2017;2017:2520510.

-30 Cankaya E, Bilen Y, Keles M, Uyanik A, Bilen N, Aydinli B: Neutrophil-lymphocyte ratio is significantly decreased in preemptive renal transplant patients. Transplant Proc 2015;47:1364-1368.

31 Libetta C, Sepe V, Esposito P, Galli F, Dal Canton A: Oxidative stress and inflammation: Implications in uremia and hemodialysis. Clin Biochem 2011;44:1189-1198.

-32 Hasselwander O, Young IS: Oxidative stress in chronic renal failure. Free Radic Res 1998;29:1-11.

-33 Lin YH, Glei D, Weinstein M, Wu SI, Chien KL: Additive value of interleukin-6 and C-reactive protein in risk prediction for all-cause and cardiovascular mortality among a representative adult cohort in Taiwan. J Formos Med Assoc 2017;10.1016/j.jfma.2017.02.002

-34 Yombi JC, Schwab PE, Thienpont E: Neutrophil-to-lymphocyte ratio (NLR) distribution shows a better kinetic pattern than C-reactive protein distribution for the follow-up of early inflammation after total knee arthroplasty. Knee Surg Sports Traumatol Arthrosc 2016;24:3287-3292.

-35 Yilmaz H, Uyfun M, Yilmaz TS, Namuslu M, Inan O, Taskin A, Cakmak M, Bilgic MA, Bavbek N, Akcay A, Kosar A: Neutrophil-lymphocyte ratio may be superior to C-reactive protein for predicting the occurrence of postmenopausal osteoporosis. Endocr Regul 2014;48:25-33.

-36 Soehnlein O: Multiple roles for neutrophils in atherosclerosis. Circ Res 2012;110:875-888.

-37 Nunez J, Minana G, Bodi V, Nunez E, Sanchis J, Husser O, Llacer A: Low lymphocyte count and cardiovascular diseases. Curr Med Chem 2011;18:3226-3233.

-38 Georgianos PI, Sarafidis PA, Lasaridis AN: Arterial stiffness: a novel cardiovascular risk factor in kidney disease patients. Curr Vasc Pharmacol 2015;13:229-238.

-39 Li P, Wang L, Liu C: Overweightness, obesity and arterial stiffness in healthy subjects: a systematic review and meta-analysis of literature studies. Postgrad Med 2017;129:224-230. 


\section{Kidney \\ Blood Pressure Research}

40 Tanaka H, Munakata M, Kawano Y, Ohishi M, Shoji T, Sugawara J, Tomiyama H, Yamashina A, Yasuda H, Sawayama T, Ozawa T: Comparison between carotid-femoral and brachial-ankle pulse wave velocity as measures of arterial stiffness. J Hypertens 2009;27:2022-2027.

41 Tanaka M, Ishii H, Aoyama T, Takahashi H, Toriyama T, Kasuga H, Takeshita K, Yoshikawa D, Amano T, Murohara T: Ankle brachial pressure index but not brachial-ankle pulse wave velocity is a strong predictor of systemic atherosclerotic morbidity and mortality in patients on maintenance hemodialysis. Atherosclerosis 2011;219:643-647.

-42 Rodriguez RA, Cronin V, Ramsay T, Zimmerman D, Ruzicka M, Burns KD: Reproducibility of carotid-femoral pulse wave velocity in end-stage renal disease patients: methodological considerations. Can J Kidney Health Dis 2016;3:20.

43 Tatar E, Sezis Demirci M, Kircelli F, Gungor O, Yaprak M, Asci G, Basci A, Ozkahya M, Ok E: The association between thyroid hormones and arterial stiffness in peritoneal dialysis patients. Int Urol Nephrol 2012;44:601-606. 